\title{
Cow's milk allergy non-responsive to amino acid-based formula? A successful transplanted patient with Immune dysregulation, polyendocrinopathy, enteropathy, X-linked syndrome
}

Liz Eliana Veramendi-Espinoza ${ }^{1}$, Claudia Alejandra Rentería-Valdivi ${ }^{1}$, Roxana Díaz-Subauste $^{1}$, Juan Carlos Aldave-Becerra ${ }^{1}$, Guisela Alva Lozada ${ }^{1}$, Marco Becilli ${ }^{2}$, and Franco Locatelli ${ }^{3}$

${ }^{1}$ Hospital Nacional Edgardo Rebagliati Martins

${ }^{2}$ Ospedale Pediatrico Bambino Gesù

${ }^{3}$ Ospedale Pediatrico Bambino Gesu

September 24, 2021

\begin{abstract}
Cow's milk allergy non-responsive to amino acid-based formula must raise suspicion of Immune dysregulation, polyendocrinopathy, enteropathy, X-linked syndrome. This is a potentially fatal disease associated with food allergy, sometimes leading to diagnostic delay. In our case, early diagnosis and management provided life-saving therapy.
\end{abstract}

\section{Hosted file}

Manuscript_08-16-21.DOCX available at https://authorea.com/users/435800/articles/538571cow-s-milk-allergy-non-responsive-to-amino-acid-based-formula-a-successful-transplantedpatient-with-immune-dysregulation-polyendocrinopathy-enteropathy-x-linked-syndrome

\section{Hosted file}

Figures_07-26-21.docx available at https://authorea.com/users/435800/articles/538571-cows-milk-allergy-non-responsive-to-amino-acid-based-formula-a-successful-transplantedpatient-with-immune-dysregulation-polyendocrinopathy-enteropathy-x-linked-syndrome 